\title{
Dinâmica populacional de doze espécies arbóreas de diferentes grupos ecológicos em um trecho de Floresta Ombrófila Densa Submontana
}

Population dynamics of twelve tree species of different ecological groups of the Submontane Ombrophilous Dense Forest

\author{
S. C. P. M. de Souza ${ }^{1 *}$; F. A. M. dos Santos 2 ; R. R. Rodrigues ${ }^{3}$; C. A. Joly ${ }^{2}$ \\ ${ }^{I}$ Seção de Madeira e Produtos Florestais, Divisão de Dasonomia, Instituto Florestal, CEP:02377-000, São Paulo- \\ SP, Brasil \\ ${ }^{2}$ Departamento de Biologia Vegetal, Instituto de Biologia, UNICAMP, CEP:13081-970, Campinas - SP, Brasil \\ ${ }^{3}$ Departamento de Ciências Biológicas, Laboratório de Ecologia e Restauração Florestal, ESALQ, CEP:13418-900, \\ Piracicaba-SP, Brasil \\ *silvana1souza@gmail.com
}

(Recebido em 16 de dezembro de 2017; aceito em 16 de maio de 2018)

\begin{abstract}
Estudos de dinâmica populacional podem contribuir na classificação das espécies em grupos ecológicos. O presente estudo teve como objetivo descrever a dinâmica populacional de 12 espécies arbóreas de diferentes grupos ecológicos e verificar se espécies com diferentes capacidades de tolerância à sombra diferem quanto à taxa de mortalidade, taxa de recrutamento e ao crescimento em altura e diâmetro. A área de estudo localiza-se na região sul do estado de São Paulo, município de Sete Barras, no interior do Parque Estadual de Carlos Botelho, em uma parcela permanente de 10 ha. Sorteamos 25 subparcelas de 20 x 20 m, onde foram realizadas duas amostragens dos indivíduos com altura $\geq 3 \mathrm{~cm}$. A taxa de mortalidade foi menor para as espécies clímax de subosque, e apenas duas espécies deste grupo apresentaram incremento populacional, Inga marginata $(0,04)$ e Rudgea jasminoides $(0,05)$, já as espécies secundárias iniciais e de dossel apresentaram um contínuo de respostas. A taxa de recrutamento e o crescimento em altura e diâmetro não diferiram entre as espécies com diferentes níveis de tolerância à sombra.

Palavras-chave: dinâmica de populações, grupos ecológicos, tolerância à sombra
\end{abstract}

Population dynamics studies can contribute to the classification of species in ecological groups. The present study aimed to describe the population dynamics of 12 tree species from different ecological groups and to verify if species with different shade tolerance capacities differ in terms of mortality rate, recruitment rate and growth in height and diameter. The study area is located in the southern region of the state of São Paulo, in the municipality of Sete Barras, within the Carlos Botelho State Park, inside a permanent plot of 10 ha. The study area is located in the Carlos Botelho State Park, inside a permanent plot of 10 ha. We sampled 25 subplots of $20 \times 20 \mathrm{~m}$, where two samples were taken from individuals with height $\geq 3 \mathrm{~cm}$. The mortality rate was lower for the understory climax species, and only two species of this group presented a population increase, Inga marginata (0.04) and Rudgea jasminoides (0.05), while the initial and canopy secondary species presented a continuous of responses. The recruitment rate and growth in height and diameter did not differ between species with different levels of shadow tolerance.

Keywords: population dynamics, ecological groups, shade-tolerance

\section{INTRODUÇÃO}

Estudos de dinâmica populacional que contemplam plântulas e plantas jovens são escassos [1, 2], apesar da importante influência dos indivíduos jovens na dinâmica populacional das espécies arbóreas de florestas tropicais [3]. Como regra geral, plantas em fases iniciais de desenvolvimento, e destas as de menor tamanho, têm taxa de mortalidade muito mais alta do que as plantas em fases maduras ou de maior tamanho [4,5].

Variações intra e interespecíficas nas taxas de crescimento, mortalidade e recrutamento são comuns nas florestas tropicais [2]. Essa variação exerce forte influência na estrutura e composição dessas florestas [6]. Embora alguns estudos sobre a dinâmica de comunidades arbóreas não 
mostrem uma clara relação de dependência entre tamanho e taxa de mortalidade [7], outros, mais recentes, apontam diferenças significativas entre grupos de espécies pertencentes a diferentes estratos da floresta $[5,8,9]$.

Grupos de espécies definidos com base no grau de tolerância à sombra podem indicar diferenças na autoecologia das espécies que apareceriam em resposta a um gradiente de luz dinâmico [10]. Tais grupos podem diferir entre si de acordo com taxas de crescimento máximo, longevidade, recrutamento e sobrevivência, assim como na sua arquitetura e capacidade fotossintética [10, 11, 12].

Segundo alguns estudos realizados em florestas tropicais conservadas, espécies secundárias iniciais apresentariam altas taxas de mortalidade e recrutamento e, espécies clímax de dossel baixas taxas mortalidade e recrutamento [5]. No entanto, ambas apresentariam um investimento em crescimento (principalmente em altura) antes de atingir a maturidade. Já as espécies clímax de subosque apresentariam baixas taxas de mortalidade, recrutamento e crescimento e formariam o único grupo composto por espécies capazes de se reproduzir sob condições de sombra $[8,10$, 13].

Dentro desta perspectiva, o presente estudo teve como objetivo analisar a dinâmica populacional de 12 espécies arbóreas com diferentes graus de tolerância à sombra (secundárias iniciais, clímax de dossel e clímax de subosque) em um trecho da Floresta Ombrófila Densa Submontana. E testar a hipótese de que as taxas de mortalidade, recrutamento e crescimento em altura e diâmetro, diferem entre espécies com diferentes capacidades de tolerância à sombra.

\section{MATERIAL E MÉTODOS}

\section{Área de estudo}

A área do estudo localiza-se no município de Sete Barras, região sul do estado de São Paulo, sobre a vertente atlântica da Serra de Paranapiacaba, no interior do Parque Estadual de Carlos Botelho (PECB) $\left(24^{\circ} 00^{\prime}\right.$ a $24^{\circ} 15^{\prime} \mathrm{S}, 47^{\circ} 45^{\prime}$ a $\left.48^{\circ} 10^{\prime} \mathrm{W}\right)$. Nessa área a vegetação é classificada como Floresta Ombrófila Densa Submontana (cota de 300 m) [14]. Localiza-se na unidade geomorfológica conhecida como Planalto de Guapiara, apresenta clima quente úmido sem estiagem, classificada como Cfa segundo o sistema de Köppen (1948) [15], com temperaturas inferiores a $18{ }^{\circ} \mathrm{C}$ no mês mais frio e superiores a $22{ }^{\circ} \mathrm{C}$ no mês mais quente. A área é caracterizada, predominantemente, por períodos de excedente hídrico, com precipitação média anual de $1582 \mathrm{~mm}$, concentrando-se nos meses de outubro a março [16].

O estudo foi conduzido no interior de uma parcela permanente de 10 ha, em uma área contínua de 320 x $320 \mathrm{~m}$ (10,24 ha), subdividida em 256 subparcelas de 20 × $20 \mathrm{~m}\left(400 \mathrm{~m}^{2}\right)$. Para este estudo, foram sorteadas 25 subparcelas, dentre as 256 dos 10 ha da Parcela Permanente, totalizando 1 ha de área amostral. Para o sorteio das subparcelas foram consideradas apenas aquelas sobre o solo Cambissolo Háplico Tb distrofico latossólico, em decorrência da sua predominância na parcela, já que os demais solos encontrados na parcela restringem-se a pequenas manchas [17].

Nas subparcelas sorteadas prevaleceram as situações de subosque e pequenas clareiras, e entre essas situações não são encontradas diferenças significativas de luminosidade [18]. Segundo Lima (2005) [19], clareiras pequenas se formam em consequência da queda de emaranhados de lianas e/ou cipós, da queda de galhos ou da desintegração gradual da copa de uma árvore morta em pé. Geralmente, a queda isolada de árvores não gera distúrbios, estes ocorrem comumente a partir de deslizamentos em encostas, furacões, ou mesmo a queda associada de várias árvores [20]. No período anterior e durante o estudo não foram observados distúrbios nas subparcelas sorteadas ou próximas a elas.

Selecionamos 12 espécies classificadas em três grupos ecológicos, segundo Brancalion et al. (2015) [21], com base na tolerância à sombra: i) secundárias iniciais, ii) clímax de dossel e iii) clímax de subosque (Tabela 1). A escolha das espécies baseou-se no levantamento fitossociológico realizado na parcela permanente do PECB [16]. Os critérios utilizados para a 
escolha das espécies foram abundância, facilidade de identificação em todos os estádios de desenvolvimento e por serem representantes dos grupos ecológicos propostos.

Para o enquadramento das espécies nos grupos ecológicos foram consultadas as características ecológicas e biológicas de cada espécie em diferentes fontes bibliográficas e observações de campo (Tabela 1). Nos casos em que ocorreu discordância entre os diversos autores ou ausência de informações sobre a classificação sucessional, prevaleceram as observações de campo.

Tabela 1. Espécies selecionadas para o estudo da dinâmica populacional no Parque Estadual de Carlos Botelho, Sete Barras, SP, com a classificação sucessional e as referências de cada classificação. A classificação final foi baseada nas categorias definidas por Brancalion et al (2015).

\begin{tabular}{|c|c|c|c|}
\hline Espécie & $\begin{array}{l}\text { Classificação } \\
\text { sucessional }\end{array}$ & Referência & Classificação final \\
\hline Alseis floribunda Schott & Secundária & [22] & Secundária inicial \\
\hline $\begin{array}{l}\text { Bathysa australis (A.St.-Hill.) } \\
\text { K.Schum. }\end{array}$ & Secundária inicial & {$[23]$} & Secundária inicial \\
\hline $\begin{array}{l}\text { Handroanthus serratifolius (Vah) } \\
\text { S.Grose }\end{array}$ & Secundária inicial & [24] & Secundária inicial \\
\hline $\begin{array}{l}\text { Myrsine hermogenesii (Jung-Mend. \& } \\
\text { Bernacci) M.F.Freitas \& Kin.-Gouv.) }\end{array}$ & Secundária inicial & {$[22]$} & Secundária inicial \\
\hline Chrysophyllum viride Mart. \& Eichler & Típica de dossel & [25] & Clímax de dossel \\
\hline Quiina glazovii Engl. & Secundária tardia & [26] & Clímax de dossel \\
\hline $\begin{array}{l}\text { Tetrastylidium grandifolium (Baill.) } \\
\text { Sleumer }\end{array}$ & Típica de dossel & [27] & Clímax de dossel \\
\hline Eugenia expansa Spring ex Mart. & Clímax & [28] & Clímax de subosque \\
\hline $\begin{array}{l}\text { Garcinia gardneriana (Planch. \& } \\
\text { Triana) Zappi }\end{array}$ & $\begin{array}{l}\text { Tolerante à sombra } \\
\text { de subosque }\end{array}$ & [29] & Clímax de subosque \\
\hline Guapira opposita (Vell.) Reitz & Tolerante à sombra & {$[28]$} & Clímax de subosque \\
\hline Inga marginata Willd. & Tolerante à sombra & [28] & Clímax de subosque \\
\hline $\begin{array}{l}\text { Rudgea jasminoides (Cham.) } \\
\text { Müll.Arg. }\end{array}$ & Tolerante à sombra & {$[28]$} & Clímax de subosque \\
\hline
\end{tabular}

Nestas unidades amostrais todos os indivíduos das espécies selecionadas, com altura $\geq 3 \mathrm{~cm}$, foram marcados com fita plástica numerada e tiveram registrado altura, utilizando-se um metro duplo para os indivíduos menores que $2 \mathrm{~m}$ e uma régua telescópica para os indivíduos maiores que $2 \mathrm{~m}$; e o diâmetro do caule no nível do solo (DAS), utilizando um paquímetro (precisão de $0,1 \mathrm{~mm}$ ) ou uma fita métrica para medir o perímetro, no caso de indivíduos de maior diâmetro.

Para a avaliação da dinâmica populacional foram realizadas duas amostragens: a primeira de outubro a dezembro de 2004 e a segunda na mesma época em 2005.

\section{Análise dos dados}

Os indivíduos de cada espécie foram divididos em classes de tamanho com base em diâmetros e alturas. O mesmo número de classes foi utilizado para todas as espécies. A divisão das classes foi realizada levando-se em consideração a amplitude de tamanho dos indivíduos, para possibilitar a comparação entre as espécies. Todos os indivíduos foram divididos em cinco classes de tamanho, com intervalos variáveis, dependendo da amplitude dos dados.

Foi calculada a taxa de mortalidade a partir do modelo exponencial contínuo [30], segundo o qual: $\mathrm{m}_{\mathrm{e}}=-\ln \left(\left(\mathrm{N}_{\mathrm{t}}-\mathrm{M}\right) / \mathrm{N}_{\mathrm{t}}\right) / \Delta \mathrm{t}$; onde $\mathbf{m}_{\mathrm{e}}$ é a taxa de mortalidade anual da população, $\mathbf{N}_{\mathrm{t}}$ é o número de indivíduos no início do período em questão, $\mathbf{M}$ é o número de indivíduos que morreram e $\Delta \mathbf{t}$ o intervalo de tempo decorrido entre as duas amostragens.

As taxas anuais de ingresso (i) também seguiram o modelo exponencial, sendo calculadas a partir da fórmula: $\mathrm{i}=\ln \left(\left(\mathrm{N}_{\mathrm{t}}+\mathrm{I}\right) / \mathrm{N}_{\mathrm{t}}\right) / \Delta \mathrm{t}$;onde $\mathbf{I}$ é o número de indivíduos que atingiu o diâmetro ou altura mínimo de cada classe de tamanho no período. 
Para verificar se houve balanceamento das taxas de mortalidade e recrutamento em cada classe de diâmetro e altura de cada espécie, foi calculada a taxa de incremento anual (r) a partir da seguinte fórmula: $\mathrm{r}=\ln \left(\left(\mathrm{N}_{\mathrm{t}}+\Delta \mathrm{t}\right) / \mathrm{N}_{\mathrm{t}}\right) / \Delta \mathrm{t}$; onde $\mathbf{N}_{\mathrm{t}}+\Delta \mathbf{t}$ é o número de indivíduos no final do período. Valores de $\mathbf{r}=0$ indicam estabilidade populacional, valores $\mathbf{r}>0$ indicam aumento da população e de $\mathbf{r}<0$ indicam declínio populacional.

Os valores de crescimento em altura e diâmetro no nível do solo (DAS) de cada indivíduo foram obtidos a partir do cálculo da diferença entre o valor medido na primeira e na segunda amostragem, dividido pelo período de tempo entre as duas medições, em anos $(\mathbf{t}=1)$. Para estes cálculos foram desconsiderados os indivíduos mortos e recrutados na segunda amostragem.

Mesmo após a transformação dos dados, as taxas de crescimento não apresentaram distribuição normal e homogeneidade de variâncias, motivo pelo qual foi empregado aos dados originais a Análise de Variância não paramétrica de Kruskal-Wallis, seguida pelo teste não paramétrico de comparações múltiplas de Dunn, para comparar as taxas de crescimento entre espécies e entre classes de tamanho. As referidas análises foram realizadas com nível de significância de $1 \%$ utilizando-se o software "R" versão 3.3.1 [31].

\section{RESULTADOS}

Foram amostrados na primeira medição 5.663 indivíduos e na segunda 5.410 indivíduos. A maioria das espécies manteve a mesma posição em número de indivíduos nos dois anos avaliados (Tabela 2).

Tabela 2. Número total de indivíduos medidos em 2004 e 2005, número de indivíduos mortos e recrutados em 2005, taxa anual de mortalidade, recrutamento e incremento populacional total das espécies estudadas no Parque Estadual de Carlos Botelho, SP, Brasil. Nt: número inicial de indivíduos em 2004, $N_{t+1}$ : número final de indivíduos em 2005, M: número de indivíduos mortos, $R$ : número de indivíduos recrutados, me: taxa anual de mortalidade, I: taxa anual de ingresso, $r$ : incremento populacional.

\begin{tabular}{lllllllc}
\hline Espécies & $\mathrm{N}_{\mathrm{t}}$ & $\mathrm{N}_{\mathrm{t}+1}$ & $\mathrm{M}$ & $\mathrm{R}$ & $\mathrm{m}_{\mathrm{e}}$ & $\mathrm{I}$ & $\mathrm{r}$ \\
\hline Secundárias iniciais & & & & & & & \\
\hline Alseis floribunda & 97 & 91 & 8 & 2 & 0,086 & 0,020 & $-0,064$ \\
\hline Bathysa australis & 129 & 124 & 8 & 3 & 0,064 & 0,023 & $-0,040$ \\
\hline Handroanthus serratifolius & 99 & 96 & 4 & 1 & 0,041 & 0,010 & $-0,031$ \\
\hline Myrsine hermogenesii & 468 & 467 & 84 & 83 & 0,198 & 0,163 & $-0,002$ \\
\hline Clímax de dossel & & & & & & & \\
\hline Chrysophyllum viride & 134 & 113 & 23 & 2 & 0,188 & 0,015 & $-0,170$ \\
\hline Quiina glaziovii & 134 & 127 & 7 & 0 & 0,054 & 0,000 & $-0,054$ \\
\hline Tetrastylidium grandifolium & 1732 & 1529 & 333 & 130 & 0,214 & 0,072 & $-0,125$ \\
\hline Clímax de sobosque & & & & & & & \\
\hline Eugenia expansa & 337 & 324 & 14 & 1 & 0,042 & 0,003 & $-0,039$ \\
\hline Garcinia gardneriana & 525 & 514 & 10 & 1 & 0,019 & 0,002 & $-0,021$ \\
\hline Guapira opposita & 988 & 957 & 53 & 22 & 0,055 & 0,022 & $-0,032$ \\
\hline Inga marginata & 377 & 393 & 44 & 60 & 0,124 & 0,148 & 0,042 \\
\hline Rudgea jasminoides & 643 & 675 & 17 & 49 & 0,027 & 0,073 & 0,049 \\
\hline
\end{tabular}

O recrutamento foi maior para Myrsine hermogenesii (16,3\%.ano $\left.{ }^{-1}\right)$, Inga marginata $\left(14,8 \%\right.$.ano $\left.{ }^{-1}\right)$ e Rudgea jasminoides $\left(7,3 \%\right.$.ano $\left.{ }^{-1}\right)$; enquanto as menores taxas de recrutamento foram de Quiina glaziovii $\left(0 \%\right.$. ano $\left.^{-1}\right)$ e Garcinia gardneriana $\left(0,2 \%\right.$. ano $\left.^{-1}\right)$. Considerando o recrutamento, não foi observado um padrão entre as espécies do mesmo grupo ecológico (Tabela 2).

As espécies com as maiores taxas de mortalidade foram Tetrastylidum grandifolium $\left(21,4 \%\right.$.ano $\left.{ }^{-1}\right)$, Myrsine hermogenesii $\left(19,8 \% \cdot\right.$ ano $\left.^{-1}\right)$ e Chrysophyllum viride $\left(18,8 \%\right.$.ano $\left.{ }^{-1}\right)$. A taxa de mortalidade foi inferior a $6 \%$ para a maioria das espécies clímax de subosque, exceto Inga 
marginata $\left(12,4 \%\right.$. ano $\left.{ }^{-1}\right)$. Para os demais grupos ecológicos não foi observado um padrão (Tabela 2). A mortalidade se concentrou na menor classe de tamanho para todas as espécies e diminuiu a partir da segunda classe de tamanho, tanto para a altura quanto para o diâmetro. Considerando a altura, a partir da terceira classe de tamanho não foi observada mortalidade (Tabela 3). Já para o diâmetro, apenas Eugenia expansa e Rudgea jasminoides apresentaram mortalidade na segunda e terceira classes, respectivamente (Tabela 4).

Tabela 3. Número total de indivíduos medidos em 2004 e 2005, número de indivíduos mortos e recrutados em 2005 e taxa anual de mortalidade, recrutamento e incremento populacional por classe de altura, no Parque Estadual Carlos Botelho, SP, Brasil. Nt: número inicial de indivíduos em 2004, $N_{t+1}$ : número final de indivíduos em 2005, M: número de indivíduos mortos, R: número de indivíduos recrutados, Ing: número de indivíduos que mudaram de classe de tamanho, me: taxa anual de mortalidade, I: taxa anual de ingresso, $r$ : incremento populacional.

\begin{tabular}{|c|c|c|c|c|c|c|c|c|}
\hline Classes de altura $(\mathrm{cm})$ & $\mathrm{N}_{\mathrm{t}}$ & $\mathrm{N}_{\mathrm{t}+1}$ & $\mathrm{M}$ & $\mathrm{R}$ & Ing & $\mathrm{m}_{\mathrm{e}}$ & $\mathrm{I}$ & $\mathrm{r}$ \\
\hline \multicolumn{9}{|l|}{ Alseis floribunda } \\
\hline $5-194$ & 86 & 79 & 8 & 2 & 0 & 0,098 & 0,052 & $-0,085$ \\
\hline$>194-572$ & 7 & 9 & 0 & 0 & 2 & 0,000 & 0,314 & 0,251 \\
\hline$>572-761$ & 2 & 2 & 0 & 0 & 0 & 0,000 & 0,000 & 0,000 \\
\hline$>761-950$ & 2 & 2 & 0 & 0 & 0 & 0,000 & 0,000 & 0,000 \\
\hline \multicolumn{9}{|l|}{ Bathysa australis } \\
\hline $2-242$ & 103 & 97 & 7 & 3 & 0 & 0,070 & 0,045 & $-0,060$ \\
\hline$>242-482$ & 11 & 11 & 1 & 0 & 1 & 0,095 & 0,226 & 0,000 \\
\hline$>482-721$ & 9 & 9 & 0 & 0 & 0 & 0,000 & 0,000 & 0,000 \\
\hline$>721-961$ & 4 & 4 & 0 & 0 & 1 & 0,000 & 0,402 & 0,000 \\
\hline$>961-1200$ & 2 & 3 & 0 & 0 & 1 & 0,000 & 0,549 & 0,405 \\
\hline \multicolumn{9}{|l|}{ Chrysophyllum viride } \\
\hline $9-510$ & 121 & 98 & 23 & 2 & 0 & 0,211 & 0,040 & $-0,211$ \\
\hline$>510-1007$ & 5 & 7 & 1 & 0 & 2 & 0,223 & 0,389 & 0,336 \\
\hline$>1007-1505$ & 6 & 4 & 0 & 0 & 0 & 0,000 & 0,000 & $-0,405$ \\
\hline$>1505-2002$ & 1 & 3 & 0 & 0 & 1 & 0,000 & 0,693 & 1,099 \\
\hline$>2002-2500$ & 1 & 1 & 0 & 0 & 0 & 0,000 & 0,000 & 0,000 \\
\hline \multicolumn{9}{|l|}{ Eugenia expansa } \\
\hline $7-186$ & 241 & 225 & 12 & 1 & 0 & 0,051 & 0,023 & $-0,069$ \\
\hline$>186-364$ & 62 & 55 & 1 & 0 & 7 & 0,016 & 0,068 & $-0,120$ \\
\hline$>364-543$ & 27 & 35 & 1 & 0 & 11 & 0,038 & 0,135 & 0,260 \\
\hline$>543-721$ & 6 & 8 & 0 & 0 & 2 & 0,000 & 0,347 & 0,288 \\
\hline$>721-900$ & 1 & 1 & 0 & 0 & 0 & 0,000 & 0,000 & 0,000 \\
\hline \multicolumn{9}{|l|}{ Garcinia gardneriana } \\
\hline $8-266$ & 362 & 346 & 9 & 1 & 0 & 0,025 & 0,016 & $-0,045$ \\
\hline$>266-525$ & 94 & 88 & 3 & 0 & 12 & 0,032 & 0,050 & $-0,066$ \\
\hline$>525-783$ & 48 & 55 & 0 & 0 & 13 & 0,000 & 0,086 & 0,136 \\
\hline$>783-1042$ & 14 & 18 & 0 & 0 & 4 & 0,000 & 0,206 & 0,251 \\
\hline$>1042-1300$ & 7 & 7 & 0 & 0 & 0 & 0,000 & 0,000 & 0,000 \\
\hline \multicolumn{9}{|l|}{ Guapira opposita } \\
\hline $3-282$ & 794 & 756 & 52 & 21 & 4 & 0,068 & 0,008 & $-0,049$ \\
\hline$>282-562$ & 165 & 164 & 1 & 0 & 12 & 0,006 & 0,031 & $-0,006$ \\
\hline$>562-841$ & 19 & 26 & 0 & 0 & 8 & 0,000 & 0,173 & 0,314 \\
\hline$>841-1121$ & 8 & 8 & 0 & 0 & 1 & 0,000 & 0,275 & 0,000 \\
\hline$>1121-1400$ & 2 & 3 & 0 & 0 & 1 & 0,000 & 0,549 & 0,405 \\
\hline \multicolumn{9}{|c|}{ Handroanthus serratifolius } \\
\hline $7-326$ & 71 & 67 & 4 & 1 & 0 & 0,058 & 0,060 & $-0,058$ \\
\hline$>326-644$ & 12 & 12 & 0 & 0 & 2 & 0,000 & 0,220 & 0,000 \\
\hline$>644-963$ & 10 & 11 & 0 & 0 & 1 & 0,000 & 0,240 & 0,095 \\
\hline$>963-1281$ & 4 & 2 & 0 & 0 & 0 & 0,000 & 0,000 & $-0,693$ \\
\hline
\end{tabular}




\begin{tabular}{lllllllcc}
\hline Classes de altura $(\mathrm{cm})$ & $\mathrm{N}_{\mathrm{t}}$ & $\mathrm{N}_{\mathrm{t}+1}$ & $\mathrm{M}$ & $\mathrm{R}$ & $\mathrm{Ing}$ & $\mathrm{m}_{\mathrm{e}}$ & $\mathrm{I}$ & $\mathrm{r}$ \\
\hline$>1281-1600$ & 2 & 4 & 0 & 0 & 2 & 0,000 & 0,693 & 0,693 \\
\hline Inga marginata & & & & & & & & \\
\hline $3-162$ & 347 & 363 & 42 & 60 & 2 & 0,129 & 0,017 & 0,045 \\
\hline$>162-322$ & 21 & 22 & 2 & 0 & 4 & 0,100 & 0,153 & 0,047 \\
\hline$>322-481$ & 4 & 3 & 0 & 0 & 0 & 0,000 & 0,000 & $-0,288$ \\
\hline$>481-641$ & 3 & 3 & 0 & 0 & 0 & 0,000 & 0,000 & 0,000 \\
\hline$>641-800$ & 2 & 2 & 0 & 0 & 0 & 0,000 & 0,000 & 0,000 \\
\hline Quiina glaziovii & & & & & & & & \\
\hline $9-267$ & 107 & 99 & 6 & 0 & 0 & 0,058 & 0,044 & $-0,078$ \\
\hline$>267-525$ & 15 & 16 & 1 & 0 & 2 & 0,069 & 0,189 & 0,065 \\
\hline$>525-784$ & 6 & 5 & 0 & 0 & 0 & 0,000 & 0,000 & $-0,182$ \\
\hline$>784-1042$ & 4 & 5 & 0 & 0 & 1 & 0,000 & 0,402 & 0,223 \\
\hline$>1042-1300$ & 2 & 2 & 0 & 0 & 0 & 0,000 & 0,000 & 0,000 \\
\hline Myrsine hermogenesii & & & & & & & & \\
\hline $4-243$ & 446 & 445 & 84 & 83 & 1 & 0,209 & 0,014 & $-0,002$ \\
\hline$>243-482$ & 8 & 8 & 0 & 0 & 1 & 0,000 & 0,275 & 0,000 \\
\hline$>482-722$ & 9 & 8 & 0 & 0 & 0 & 0,000 & 0,000 & $-0,118$ \\
\hline$>722-961$ & 3 & 3 & 0 & 0 & 1 & 0,000 & 0,462 & 0,000 \\
\hline$>961-1200$ & 2 & 3 & 0 & 0 & 1 & 0,000 & 0,549 & 0,405 \\
\hline Rudgea jasminoides & & & & & & & & \\
\hline $3-162$ & 605 & 638 & 16 & 49 & 0 & 0,027 & 0,011 & 0,053 \\
\hline$>162-481$ & 16 & 12 & 1 & 0 & 0 & 0,065 & 0,000 & $-0,288$ \\
\hline$>481-641$ & 15 & 15 & 0 & 0 & 3 & 0,000 & 0,193 & 0,000 \\
\hline$>641-800$ & 7 & 10 & 0 & 0 & 3 & 0,000 & 0,329 & 0,357 \\
\hline Tetrastylidium grandifolium & & & & & & & & \\
\hline 5-404 & 1682 & 1464 & 333 & 130 & 0 & 0,221 & 0,004 & $-0,139$ \\
\hline$>404-803$ & 21 & 35 & 1 & 0 & 15 & 0,049 & 0,171 & 0,511 \\
\hline$>803-1202$ & 20 & 20 & 0 & 0 & 1 & 0,000 & 0,152 & 0,000 \\
\hline$>1202-1601$ & 8 & 6 & 0 & 0 & 1 & 0,000 & 0,275 & $-0,288$ \\
\hline$>1601-2000$ & 1 & 4 & 0 & 0 & 3 & 0,000 & 1,386 & 1,386 \\
\hline & & & & & & & &
\end{tabular}

Apenas duas espécies apresentaram incremento populacional, ambas clímax de subosque, Inga marginata, e Rudgea jasminoides (Tabela 2) O decréscimo populacional foi verificado para a totalidade das espécies clímax de dossel e secundárias iniciais, as espécies com as maiores taxas de decréscimo populacional foram Chrysophyllum viride e Tetrastylidium grandifolium (Tabela 2).

O crescimento em altura e diâmetro foi maior com o aumento da classe de tamanho para todas as espécies clímax de dossel e clímax de subosque; estes grupos apresentaram diferenças de crescimento entre a menor classe e as demais (Tabelas 5 e 6). Nestes casos, a média de crescimento foi maior nos indivíduos pertencentes às maiores classes de tamanho. As espécies secundárias iniciais, o crescimento em altura não diferiu entre as classes (Tabela 5), exceto Handroanthus serratifolius, já o crescimento em diâmetro, apenas Alseis floribunda não diferiu entre a menor classe de tamanho e as demais classes (Tabela 6). 
Tabela 4. Número total de indivíduos medidos em 2004 e 2005, número de indivíduos mortos e recrutados em 2005 e taxa anual de mortalidade, recrutamento e incremento populacional por classe de diâmetro (DAS), no Parque Estadual Carlos Botelho, SP, Brasil. Nt: número inicial de indivíduos em

2004, $N_{t+1}$ : número final de indivíduos em 2005, M: número de indivíduos mortos, $R$ : número de indivíduos recrutados, Ing: número de indivíduos que mudaram de classe de tamanho, me: taxa anual de mortalidade, I: taxa anual de ingresso, r: incremento populacional.

\begin{tabular}{|c|c|c|c|c|c|c|c|c|}
\hline Classes de DAS & $\mathrm{N}_{\mathrm{t}}$ & $\mathrm{N}_{\mathrm{t}+1}$ & $\mathrm{M}$ & $\mathrm{R}$ & Ing & $\mathrm{m}_{\mathrm{e}}$ & $\mathrm{I}$ & $\mathrm{r}$ \\
\hline \multicolumn{9}{|l|}{ Alseis floribunda } \\
\hline $0,1-3,6$ & 84 & 78 & 8 & 2 & 0 & 0,100 & 0,053 & $-0,074$ \\
\hline$>3,6-7,1$ & 9 & 9 & 0 & 0 & 0 & 0,000 & 0,000 & 0,000 \\
\hline$>7,1-14,0$ & 2 & 2 & 0 & 0 & 0 & 0,000 & 0,000 & 0,000 \\
\hline$>14,0-17,5$ & 2 & 2 & 0 & 0 & 0 & 0,000 & 0,000 & 0,000 \\
\hline \multicolumn{9}{|l|}{ Bathysa australis } \\
\hline $0,1-6,8$ & 116 & 110 & 8 & 3 & 0 & 0,071 & 0,041 & $-0,053$ \\
\hline$>6,8-13,5$ & 4 & 5 & 0 & 0 & 1 & 0,000 & 0,402 & 0,223 \\
\hline$>13,5-20,3$ & 3 & 3 & 0 & 0 & 0 & 0,000 & 0,000 & 0,000 \\
\hline$>20,3-27,0$ & 2 & 1 & 0 & 0 & 0 & 0,000 & 0,000 & $-0,693$ \\
\hline$>27,0-33,7$ & 4 & 5 & 0 & 0 & 1 & 0,000 & 0,402 & 0,223 \\
\hline \multicolumn{9}{|c|}{ Chrysophyllum viride } \\
\hline $0,1-17,3$ & 125 & 104 & 8 & 3 & 0 & 0,066 & 0,039 & $-0,184$ \\
\hline$>17,3-34,4$ & 1 & 1 & 0 & 0 & 0 & 0,000 & 0,000 & 0,000 \\
\hline$>34,4-51,6$ & 3 & 3 & 0 & 0 & 0 & 0,000 & 0,000 & 0,000 \\
\hline$>51,6-68,7$ & 2 & 2 & 0 & 0 & 0 & 0,000 & 0,000 & 0,000 \\
\hline$>68,7-85,9$ & 3 & 3 & 0 & 0 & 0 & 0,000 & 0,000 & 0,000 \\
\hline \multicolumn{9}{|l|}{ Eugenia expansa } \\
\hline $0,1-2,8$ & 274 & 262 & 13 & 1 & 1 & 0,049 & 0,020 & $-0,045$ \\
\hline$>2,8-5,5$ & 40 & 39 & 1 & 0 & 2 & 0,025 & 0,093 & $-0,025$ \\
\hline$>5,5-8,3$ & 14 & 14 & 0 & 0 & 1 & 0,000 & 0,193 & 0,000 \\
\hline$>8,3-11,0$ & 5 & 5 & 0 & 0 & 0 & 0,000 & 0,000 & 0,000 \\
\hline$>11,0-13,7$ & 4 & 4 & 0 & 0 & 0 & 0,000 & 0,000 & 0,000 \\
\hline \multicolumn{9}{|c|}{ Garcinia gardneriana } \\
\hline $0,2-6,9$ & 453 & 438 & 12 & 1 & 0 & 0,027 & 0,014 & $-0,034$ \\
\hline$>6,9-13,6$ & 46 & 47 & 0 & 0 & 4 & 0,000 & 0,085 & 0,022 \\
\hline$>13,6-20,3$ & 23 & 24 & 0 & 0 & 3 & 0,000 & 0,142 & 0,043 \\
\hline$>20,3-27,0$ & 2 & 4 & 0 & 0 & 2 & 0,000 & 0,693 & 0,693 \\
\hline$>27,0-33,7$ & 1 & 1 & 0 & 0 & 0 & 0,000 & 0,000 & 0,000 \\
\hline \multicolumn{9}{|l|}{ Guapira opposita } \\
\hline $0,2-8,8$ & 910 & 874 & 53 & 22 & 2 & 0,060 & 0,008 & $-0,040$ \\
\hline$>8,8-17,4$ & 60 & 65 & 0 & 0 & 7 & 0,000 & 0,070 & 0,080 \\
\hline$>17,4-26,1$ & 11 & 11 & 0 & 0 & 0 & 0,000 & 0,000 & 0,000 \\
\hline$>26,1-34,7$ & 4 & 4 & 0 & 0 & 0 & 0,000 & 0,000 & 0,000 \\
\hline$>34,7-43,4$ & 3 & 3 & 0 & 0 & 0 & 0,000 & 0,000 & 0,000 \\
\hline \multicolumn{9}{|c|}{ Handroanthus serratifolius } \\
\hline $0,2-11,0$ & 83 & 79 & 4 & 1 & 0 & 0,049 & 0,053 & $-0,049$ \\
\hline$>11,0-21,8$ & 11 & 12 & 0 & 0 & 1 & 0,000 & 0,226 & 0,087 \\
\hline$>21,8-32,5$ & 1 & 1 & 0 & 0 & 0 & 0,000 & 0,000 & 0,000 \\
\hline$>32,5-43,3$ & 3 & 3 & 0 & 0 & 0 & 0,000 & 0,000 & 0,000 \\
\hline$>43,3-54,1$ & 1 & 1 & 0 & 0 & 0 & 0,000 & 0,000 & 0,000 \\
\hline \multicolumn{9}{|l|}{ Inga marginata } \\
\hline $0,1-2,8$ & 367 & 380 & 44 & 60 & 0 & 0,128 & 0,017 & 0,035 \\
\hline$>2,8-8,1$ & 7 & 10 & 0 & 0 & 3 & 0,000 & 0,329 & 0,357 \\
\hline$>8,1-10,7$ & 1 & 1 & 0 & 0 & 0 & 0,000 & 0,000 & 0,000 \\
\hline$>10,7-13,4$ & 2 & 2 & 0 & 0 & 0 & 0,000 & 0,000 & 0,000 \\
\hline
\end{tabular}




\begin{tabular}{llllllllc}
\hline Classes de DAS & $\mathrm{N}_{\mathrm{t}}$ & $\mathrm{N}_{\mathrm{t}+1}$ & $\mathrm{M}$ & $\mathrm{R}$ & $\mathrm{Ing}$ & $\mathrm{m}_{\mathrm{e}}$ & $\mathrm{I}$ & $\mathrm{r}$ \\
\hline Quiina glaziovii & & & & & & & & \\
\hline $0,4-9,0$ & 125 & 117 & 7 & 0 & 0 & 0,058 & 0,000 & $-0,066$ \\
\hline$>9,0-17,6$ & 3 & 4 & 0 & 0 & 2 & 0,000 & 0,536 & 0,288 \\
\hline$>17,6-26,1$ & 4 & 3 & 0 & 0 & 1 & 0,000 & 0,402 & $-0,288$ \\
\hline$>26,1-34,7$ & 1 & 2 & 0 & 0 & 0 & 0,000 & 0,000 & 0,693 \\
\hline$>34,7-43,3$ & 1 & 1 & 0 & 0 & 2 & 0,000 & 1,099 & 0,000 \\
\hline Myrsine hermogenesii & & & & & & & & \\
\hline $0,1-6,1$ & 456 & 454 & 84 & 83 & 0 & 0,204 & 0,014 & $-0,004$ \\
\hline$>6,1-12,1$ & 3 & 4 & 0 & 0 & 1 & 0,000 & 0,462 & 0,288 \\
\hline$>12,1-18,2$ & 2 & 2 & 0 & 0 & 0 & 0,000 & 0,000 & 0,000 \\
\hline$>18,2-24,2$ & 6 & 6 & 0 & 0 & 0 & 0,000 & 0,000 & 0,000 \\
\hline$>24,2-30,2$ & 1 & 1 & 0 & 0 & 0 & 0,000 & 0,000 & 0,000 \\
\hline Rudgea jasminoides & & & & & & & & \\
\hline $0,1-4,2$ & 606 & 639 & 16 & 1 & 49 & 0,027 & 0,011 & 0,053 \\
\hline$>4,2-8,2$ & 5 & 3 & 0 & 0 & 0 & 0,000 & 0,000 & $-0,511$ \\
\hline$>8,2-12,3$ & 15 & 15 & 1 & 0 & 2 & 0,069 & 0,189 & 0,000 \\
\hline$>12,3-16,3$ & 15 & 14 & 0 & 0 & 1 & 0,000 & 0,185 & $-0,069$ \\
\hline$>16,3-20,4$ & 2 & 4 & 0 & 0 & 2 & 0,000 & 0,693 & 0,693 \\
\hline Tetrastylidium grandifolium & & & & & & & & \\
\hline $0,2-11,7$ & 1693 & 1490 & 333 & 130 & 0 & 0,219 & 0,004 & $-0,128$ \\
\hline$>11,7-23,2$ & 18 & 18 & 0 & 0 & 0 & 0,000 & 0,000 & 0,000 \\
\hline$>23,3-34,6$ & 14 & 14 & 0 & 0 & 0 & 0,000 & 0,000 & 0,000 \\
\hline$>34,6-46,1$ & 4 & 4 & 0 & 0 & 0 & 0,000 & 0,000 & 0,000 \\
\hline$>46,1-57,6$ & 3 & 3 & 0 & 0 & 0 & 0,000 & 0,000 & 0,000 \\
\hline & & & & & & & & \\
\hline & & & 14 & & & & &
\end{tabular}

Tabela 5. Análise de variância não-paramétrica de Kruskal-Wallis e teste de comparações múltiplas de Dunn das taxas anuais de crescimento em altura (cm.ano ${ }^{-1}$ ) em diferentes classes de tamanho (altura, cm) das populações estudadas no Parque Estadual Carlos Botelho, SP, Brasil. No teste de comparações múltiplas, as classes de tamanho foram arranjadas em ordem crescente e numeradas, as classes citadas foram as que apresentaram diferenças significativas.

\begin{tabular}{|c|c|c|}
\hline Espécie & $\begin{array}{l}\text { Kruskal-Wallis (H); } \\
\text { Probabilidade }\end{array}$ & $\begin{array}{c}\text { Teste de Dunn } \\
\text { (classes de altura com } \\
\text { diferenças significativas) }\end{array}$ \\
\hline Alseis floribunda & $\mathrm{H}=1,86 ; \mathrm{P}=0,1720$ & - \\
\hline Bathysa australis & $\mathrm{H}=10,06 ; \mathrm{P}=0,0394$ & - \\
\hline Chrysophyllum viride & $\mathrm{H}=16,88 ; \mathrm{P}=0,0007$ & $1 \mathrm{e} 4$ \\
\hline Eugenia expansa & $\mathrm{H}=49,59 ; \mathrm{P}=0,0000$ & 1 e $2 ; 1$ e $3 ; 1$ e 4 \\
\hline Garcina gardneriana & $\mathrm{H}=74,14 ; \mathrm{P}=0,0000$ & 1 e $2 ; 1$ e $3 ; 1$ e $4 ; 1$ e 5 \\
\hline Guapira opposita & $\mathrm{H}=100,08 ; \mathrm{P}=0,0000$ & 1 e $2 ; 1$ e $3 ; 1$ e 5 \\
\hline Handroanthus serratifolius & $\mathrm{H}=24,69 ; \mathrm{P}=0,0000$ & 1 e $2 ; 1$ e $3 ; 1$ e 5 \\
\hline Inga marginata & $\mathrm{H}=25,41 ; \mathrm{P}=0,0000$ & 1 e $2 ; 1$ e 4 \\
\hline Quiina glaziovii & $\mathrm{H}=25,41 ; \mathrm{P}=0,0000$ & $1 \mathrm{e} 2 ; 1 \mathrm{e} 4$ \\
\hline Myrsine hermogenesii & $\mathrm{H}=10,32 ; \mathrm{P}=0,0353$ & - \\
\hline Rudgea jasminoides & $\mathrm{H}=45,78 ; \mathrm{P}=0,0000$ & 1 e $2 ; 1$ e 3 \\
\hline Tetrastylidium grandifolium & $\mathrm{H}=118,16 ; \mathrm{P}=0,0000$ & 1 e $2 ; 1$ e $3 ; 1$ e $4 ; 1$ e 5 \\
\hline
\end{tabular}


Tabela 6. Análise de variância não-paramétrica de Kruskal-Wallis e teste de comparações múltiplas de Dunn das taxas anuais de crescimento em diâmetro (cm.ano ${ }^{-1}$ ) em diferentes classes de tamanho das populações estudadas no Parque Estadual Carlos Botelho, SP, Brasil. No teste de comparações múltiplas, as classes de tamanho foram arranjadas em ordem crescente e numeradas, as classes citadas foram as que apresentaram diferenças significativas.

Espécie Kruskal-Wallis $(\mathrm{H})$;

Teste de Dunn Probabilidade (classes de tamanho com diferenças significativas)

\begin{tabular}{|c|c|c|}
\hline Alseis floribunda & $\mathrm{H}=0,33 ; \mathrm{P}=0,5651$ & - \\
\hline Bathysa australis & $\mathrm{H}=25,26 ; \mathrm{P}=0,0000$ & 1 e $2 ; 1$ e 5 \\
\hline Chrysophyllum viride & $\mathrm{H}=12,16 ; \mathrm{P}=0,0023$ & 1 e 3 \\
\hline Eugenia expansa & $\mathrm{H}=40,52 ; \mathrm{P}=0,0000$ & 1 e $2 ; 1$ e 3 \\
\hline Garcina gardneriana & $\mathrm{H}=139,98 ; \mathrm{P}=0,0000$ & 1 e $2 ; 1$ e $3 ; 1$ e 4 \\
\hline Guapira opposita & $\mathrm{H}=134,61 ; \mathrm{P}=0,0000$ & 1 e $2 ; 1$ e 3 \\
\hline Handroanthus serratifolius & $\mathrm{H}=16,62 ; \mathrm{P}=0,0002$ & 1 e $2 ; 1$ e 4 \\
\hline Inga marginata & $\mathrm{H}=11,51 ; \mathrm{P}=0,0007$ & $1 \mathrm{e} 2$ \\
\hline Quiina glaziovii & $\mathrm{H}=17,95 ; \mathrm{P}=0,0001$ & 1 e $2 ; 1$ e 3 \\
\hline Myrsine hermogenesii & $\mathrm{H}=26,54 ; \mathrm{P}=0,0000$ & $1 \mathrm{e} 2 ; 1 \mathrm{e} 4$ \\
\hline Rudgea jasminoides & $\mathrm{H}=91,74 ; \mathrm{P}=0,0000$ & 1 e $2 ; 1$ e $3 ; 1$ e $4 ; 1$ e 5 \\
\hline Tetrastylidium grandifolium & $\mathrm{H}=84,62 ; \mathrm{P}=0,0000$ & 1 e $2 ; 1$ e $3 ; 1$ e $4 ; 1$ e 5 \\
\hline
\end{tabular}

Embora na análise do crescimento médio em altura entre as diferentes classes de tamanho tenha sido possível distinguir as espécies do grupo das secundárias iniciais das demais, nenhum padrão ficou evidente na comparação do crescimento médio anual das espécies (Figuras 1 e 2). Tanto para o crescimento médio em altura quanto em diâmetro, nenhuma espécie esteve muito acima da média, mas o desvio padrão foi alto para todas as espécies, principalmente quanto ao crescimento em altura (Figuras 1 e 2).

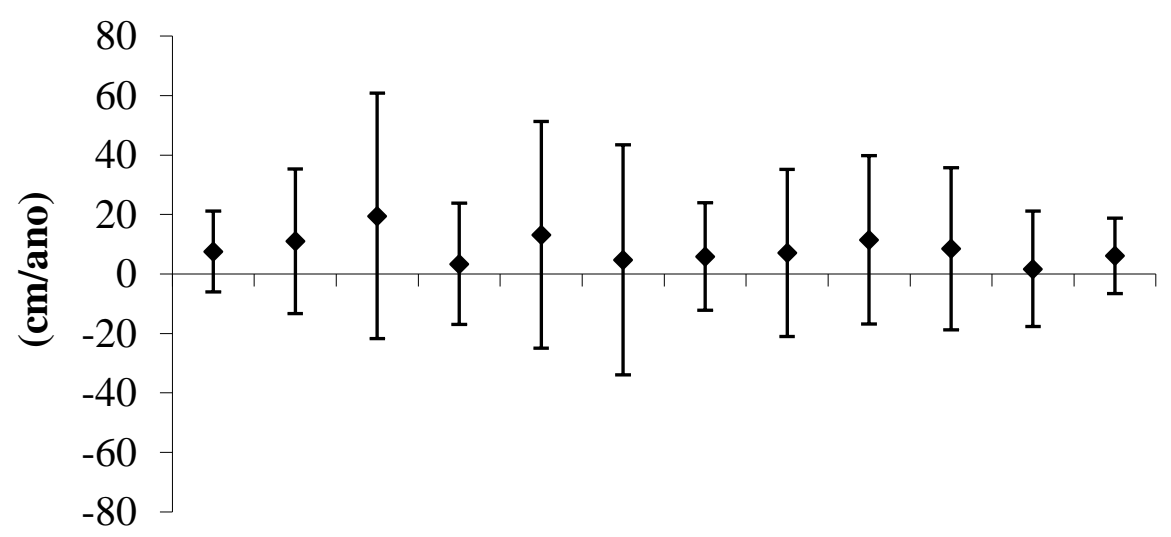

Af $\mathrm{Ba} \mathrm{Hs}$ Mh $\mathrm{Cv}$ Qg $\mathrm{Tg}$ Ee $\mathrm{Gg}$ Go $\mathrm{Im} \mathrm{Rj}$

\section{Espécies}

Figura 1. Média de crescimento anual $\left(\mathrm{cm} . a n o^{-1}\right)$ e desvio padrão da altura das populações estudadas no Parque Estadual Carlos Botelho, SP, Brasil. Af: Alseis floribunda, Ba: Bathysa australis, Cv: Chrysophyllum viride, Ee: Eugenia expansa, Gg: Garcinia gardneriana, Go: Guapira opposita, Hs: Handroanthus serratifolius, Im: Inga marginata, Qg: Quiina glaziovii, Mh: Myrsine hermogenesii, Rj: Rudgea jasminoides, Tg: Tetrastylidium grandifolium. 


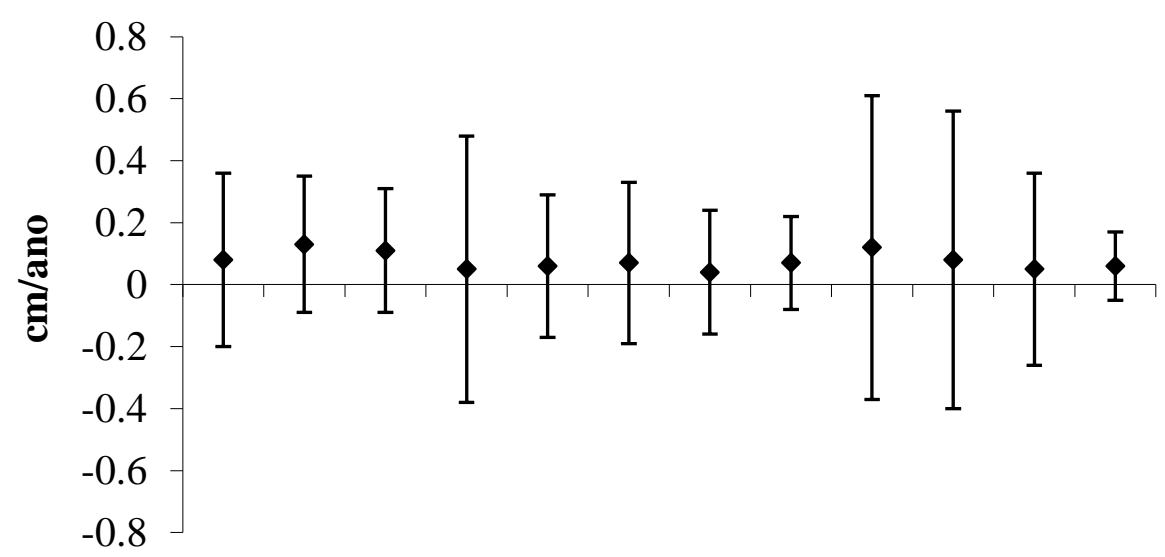

Af $\mathrm{Ba} \quad \mathrm{Hs} \quad \mathrm{Mh} \quad \mathrm{Cv} \quad \mathrm{Qg} \quad \mathrm{Tg}$ Ee $\mathrm{Gg}$ Go $\mathrm{Im} \quad \mathrm{Rj}$

Espécies

Figura 2. Média de crescimento anual ( $\left.\mathrm{cm} . a n o^{-1}\right)$ e desvio padrão do diâmetro no nível do solo das populações estudadas no Parque Estadual Carlos Botelho, SP, Brasil. Af: Alseis floribunda, Ba: Bathysa australis, Cv: Chrysophyllum viride, Ee: Eugenia expansa, Gg: Garcinia gardneriana, Go: Guapira opposita, Hs: Handroanthus serratifolius, Im: Inga marginata, Qg: Quiina glaziovii, Mh: Myrsine hermogenesii, Rj: Rudgea jasminoides, Tg: Tetrastylidium grandifolium.

Dentre as espécies secundárias iniciais, Handroanthus serratifolius, apresentou o maior crescimento médio em altura e Myrsine hermogenesii o menor crescimento, $M$. hermogenesii diferiu de Bathysa autralis e H. serratifolius. O crescimento em altura não diferiu entre as espécies de dossel. Por outro lado, entre as espécies clímax de subosque foram encontradas diferenças significativas no crescimento em altura de Inga marginata que apresentou o menor crescimento médio e diferiu de Eugenia expansa, Garcinia gardneriana e Guapira opposita. As demais espécies clímax de subosque não diferiram quanto ao crescimento em altura (Tabela 7, Figura 1).

Tabela 7. Análise de variância não-paramétrica de Kruskal-Wallis e teste de comparações múltiplas de Dunn das taxas anuais de crescimento em altura (cm.ano ${ }^{-1}$ ) entre as populações estudadas no Parque Estadual Carlos Botelho, SP, Brasil. As classes de tamanho foram arranjadas em ordem crescente e numeradas e indicadas as que apresentaram diferenças significativas. Af: Alseis floribunda, Ba: Bathysa australis, Cv: Chrysophyllum viride, Ee: Eugenia expansa, Gg: Garcinia gardneriana, Go:

Guapira opposita, Hs: Handroanthus serratifolius, Im: Inga marginata, Qg: Quiina glaziovii, Mh:

\begin{tabular}{lccccccccccc}
\multicolumn{7}{c}{ Myrsine hermogenesii, Rj: Rudgea jasminoides, Tg: Tetrastylidium grandifolium. } \\
\hline $\mathrm{Af}$ & $\mathrm{ns}$ & $\mathrm{ns}$ & $\mathrm{ns}$ & $\mathrm{ns}$ & $\mathrm{ns}$ & $\mathrm{ns}$ & $\mathrm{ns}$ & $\mathrm{ns}$ & $\mathrm{ns}$ & $\mathrm{ns}$ & $\mathrm{ns}$ \\
\hline $\mathrm{Ba}$ & - & $\mathrm{ns}$ & $\mathrm{ns}$ & $\mathrm{ns}$ & $\mathrm{ns}$ & $<0,05$ & $\mathrm{~ns}$ & $<0,05$ & $<0,05$ & $<0,05$ & $\mathrm{~ns}$ \\
\hline $\mathrm{Cv}$ & - & - & $\mathrm{ns}$ & $\mathrm{ns}$ & $\mathrm{ns}$ & $\mathrm{ns}$ & $\mathrm{ns}$ & $<0,05$ & $\mathrm{~ns}$ & $\mathrm{~ns}$ & $\mathrm{~ns}$ \\
\hline $\mathrm{Ee}$ & - & - & - & $\mathrm{ns}$ & $\mathrm{ns}$ & $<0,05$ & $\mathrm{~ns}$ & $<0,05$ & $\mathrm{~ns}$ & $<0,05$ & $\mathrm{~ns}$ \\
\hline $\mathrm{Gg}$ & - & - & - & - & $\mathrm{ns}$ & $<0,05$ & $\mathrm{~ns}$ & $<0,05$ & $\mathrm{~ns}$ & $<0,05$ & $\mathrm{~ns}$ \\
\hline $\mathrm{Go}$ & - & - & - & - & - & $<0,05$ & $\mathrm{~ns}$ & $<0,05$ & $\mathrm{~ns}$ & $<0,05$ & $\mathrm{~ns}$ \\
\hline $\mathrm{Im}$ & - & - & - & - & - & - & $\mathrm{ns}$ & $\mathrm{ns}$ & $<0,05$ & $\mathrm{~ns}$ & $\mathrm{~ns}$ \\
\hline $\mathrm{Qg}$ & - & - & - & - & - & - & - & $<0,05$ & $\mathrm{~ns}$ & $\mathrm{~ns}$ & $\mathrm{~ns}$ \\
\hline $\mathrm{Mh}$ & - & - & - & - & - & - & - & - & $<0,05$ & $\mathrm{~ns}$ & $<0,05$ \\
\hline $\mathrm{Rj}$ & - & - & - & - & - & - & - & - & - & $<0,05$ & $\mathrm{~ns}$ \\
\hline $\mathrm{Tg}$ & - & - & - & - & - & - & - & - & - & - & $\mathrm{ns}$ \\
\hline
\end{tabular}


Considerando o incremento em diâmetro dentre as espécies secundárias iniciais o maior incremento foi de Bathysa australis que diferiu de Alseis floribunda e Myrsine hermogenesii. Entre as espécies clímax de dossel o maior valor foi de Quiina glaziovii que diferiu de Tetrastylidium grandifolium. Entre as espécies clímax de subosque, não ocorreu um padrão, algumas espécies diferiram, enquanto outras não. (Tabela 8, Figura 2).

Tabela 8. Análise de variância não-paramétrica de Kruskal-Wallis e teste de comparações múltiplas de Dunn das taxas anuais de crescimento em diâmetro (cm.ano-1) entre as populações estudadas no Parque Estadual Carlos Botelho, SP, Brasil. Af: Alseis floribunda, Ba: Bathysa australis, Cv: Chrysophyllum viride, Ee: Eugenia expansa, Gg: Garcinia gardneriana, Go: Guapira opposita, Hs: Handroanthus serratifolius, Im: Inga marginata, Qg: Quiina glaziovii, Mh: Myrsine hermogenesii, Rj: Rudgea jasminoides, Tg: Tetrastylidium grandifolium.

\begin{tabular}{lcccccccccccc}
\hline & $\mathrm{Ba}$ & $\mathrm{Cv}$ & $\mathrm{Ee}$ & $\mathrm{Gg}$ & $\mathrm{Go}$ & $\mathrm{Im}$ & $\mathrm{Qg}$ & $\mathrm{Mh}$ & $\mathrm{Rj}$ & $\mathrm{Tg}$ & $\mathrm{Hs}$ \\
\hline $\mathrm{Af}$ & $<0,05$ & $\mathrm{~ns}$ & $\mathrm{~ns}$ & $<0,05$ & $<0,05$ & $\mathrm{~ns}$ & $\mathrm{~ns}$ & $\mathrm{~ns}$ & $\mathrm{~ns}$ & $\mathrm{~ns}$ & $<0,05$ \\
\hline $\mathrm{Ba}$ & - & $\mathrm{ns}$ & $\mathrm{ns}$ & $\mathrm{ns}$ & $\mathrm{ns}$ & $<0,05$ & $\mathrm{~ns}$ & $<0,05$ & $<0,05$ & $<0,05$ & $\mathrm{~ns}$ \\
\hline $\mathrm{Cv}$ & - & - & $\mathrm{ns}$ & $<0,05$ & $<0,05$ & $\mathrm{Ns}$ & $\mathrm{ns}$ & $\mathrm{ns}$ & $\mathrm{ns}$ & $\mathrm{ns}$ & $\mathrm{ns}$ \\
\hline $\mathrm{Ee}$ & - & - & - & $<0,05$ & $<0,05$ & $<0,05$ & $\mathrm{~ns}$ & $<0,05$ & $\mathrm{~ns}$ & $<0,05$ & $\mathrm{~ns}$ \\
\hline $\mathrm{Gg}$ & - & - & - & - & $\mathrm{ns}$ & $<0,05$ & $\mathrm{~ns}$ & $<0,05$ & $<0,05$ & $<0,05$ & $\mathrm{~ns}$ \\
\hline $\mathrm{Go}$ & - & - & - & - & - & $<0,05$ & $\mathrm{~ns}$ & $<0,05$ & $<0,05$ & $<0,05$ & $\mathrm{~ns}$ \\
\hline $\mathrm{Im}$ & - & - & - & - & - & - & $<0,05$ & $\mathrm{~ns}$ & $\mathrm{~ns}$ & $\mathrm{~ns}$ & $<0,05$ \\
\hline $\mathrm{Qg}$ & - & - & - & - & - & - & - & $<0,05$ & $\mathrm{~ns}$ & $<0,05$ & $\mathrm{~ns}$ \\
\hline $\mathrm{Mh}$ & - & - & - & - & - & - & - & - & $\mathrm{ns}$ & $\mathrm{ns}$ & $<0,05$ \\
\hline $\mathrm{Rj}$ & - & - & - & - & - & - & - & - & - & $<0,05$ & $<0,05$ \\
\hline $\mathrm{Tg}$ & - & - & - & - & - & - & - & - & - & - & $<0,05$ \\
\hline
\end{tabular}

\section{DISCUSSÃO}

A alta concentração de mortalidade na menor classe de tamanho obtida neste estudo segue o padrão esperado para espécies tropicais $[4,5,31,32]$. As florestas tropicais maduras apresentam predominantemente em seu interior condições de pouca luminosidade [8], nestas circunstâncias é comum entre as espécies intolerantes à sombra taxas de mortalidade superiores a 10\%, no presente estudo, dentre as secundárias iniciais, apenas Myrsine hermogenesii apresentou mortalidade superior a $10 \%$, as demais espécies do referido grupo apresentaram taxas de mortalidade inferiores a $10 \%$, no entanto, superiores ao recrutamento, resultando em decréscimo populacional.

O grupo das espécies clímax de dossel apresentaram um gradiente de respostas, quanto a mortalidade e o recrutamento, decorrente da diversidade de estratégias presente neste grupo [33, 34]. As espécies Chrysophyllum viride e Tetrastylidium grandifolium apresentaram altas taxas de mortalidade acompanhadas de altas taxas de recrutamento, já a população de Quiina glaziovii, se manteve praticamente estacionada, pois apresentou baixa mortalidade associada a ausência de recrutamento no período.

As espécies clímax de subosque apresentaram as menores taxas de mortalidade. Em estudos realizados com espécies de subosque em florestas tropicais, tem sido comum encontrar, entre as espécies tolerantes à sombra, taxas de mortalidade próximas de 2\% [35]. Taxas de sobrevivência superiores a $90 \%$ são relativamente altas, sugerindo o status de espécies não pioneiras ou tolerantes à sombra [35, 36], fortalecendo a classificação realizada a priori das referidas espécies. Dentre as espécies clímax de subosque, apenas I. marginata, apresentou alta taxa de mortalidade, no entanto a taxa de recrutamento no período também foi elevada, resultando no seu incremento populacional.

Seguindo o padrão que tem sido observado em florestas tropicais, o recrutamento não foi suficiente para contrabalançar a mortalidade da maioria das espécies estudadas $[4,5]$. As principais causas de morte em uma população de plântulas e indivíduos jovens, na maioria das vezes são dependentes da distância e densidade em relação às plantas matrizes, causadas ora pela presença de patógenos, herbívoros, alelopatia, ou ainda pela competição entre plântulas ou entre plântulas e indivíduos adultos [1]. Howe (1990) [37] apontou ainda a seca, a predação e sobretudo 
a baixa disponibilidade de luz, como principais fatores que afetaram o recrutamento de Virola surinamensis Warb. em um estudo realizado no Panamá.

A mortalidade foi maior do que o recrutamento para dez das doze espécies estudadas. Esse fato pode estar associado ao curto período analisado, que não possibilitou observar o período pósreprodutivo da maioria das espécies (Alseis floribunda, Chrysophyllum viride, Eugenia expansa, Garcinia gardneriana, Guapira opposita, Quiina glaziovii e Handroanthus serratifolius). Muitas espécies tropicais apresentam reprodução supra-anual, com intervalos irregulares [32]. Essa possível irregularidade somente é revelada após estudos de longa duração [1]. As causas implícitas deste fenômeno podem ser uma resposta às condições climáticas ou a estratégias reprodutivas [38].

Das espécies em que foi observado o período pós-reprodutivo, Bathysa australis, Inga marginata, Myrsine hermogenesii, Rudgea jasminoides e Tetrastylidium grandifolium, apenas duas apresentaram incremento populacional, I. marginata e $R$. jasminoides, ambas clímax de subosque. Para as espécies $B$. australis, M. hermogenesii e $T$. grandifolium a mortalidade foi superior ao recrutamento.

O crescimento nulo, tanto em diâmetro, como em altura é uma característica de espécies de crescimento lento, tolerantes à sombra [7]. Plântulas e jovens de espécies típicas de subosque, sob condições de baixa luminosidade, destacam-se por apresentar habilidades para sobreviver nesses ambientes, frequentemente com baixo crescimento [39]. A ausência de incremento neste estádio reflete a capacidade dos indivíduos sobreviverem a longos períodos de supressão formando um banco de plântulas e indivíduos jovens $[34,40]$. Taxas de crescimento nas florestas tropicais evidenciam uma considerável variação intra e interespecífica [2]. Em estudos demográficos, esta variação é frequentemente negligenciada ou considerada ao acaso [41]. A variação nas taxas de crescimento intraespecífica, entretanto, não é distribuída ao acaso, mas sim, consequência de características genéticas, associadas aos fatores ambientais que fazem com que algumas árvores apresentem melhor performance do que outras [42].

A importância relativa de diferentes componentes que contribuem para essa variação é difícil de investigar [38]. Muitos estudos em florestas tropicais revelam uma relação inversamente proporcional, sob baixa luminosidade, entre o crescimento e a tolerância à sombra [8,43]. Neste estudo, o incremento em altura e diâmetro não pode ser relacionado com a tolerância à sombra, uma vez que apenas entre as espécies clímax de dossel não houve diferenças significativas, rejeitando-se, desta forma, a hipótese de que o crescimento em altura e diâmetro diferem entre as espécies com diferentes níveis de tolerância à sombra. Clark \& Clark (2001) [2] concluíram, a partir de um estudo na Costa Rica, que 16 anos de medição foram insuficientes para determinar o padrão de crescimento das árvores, sendo necessárias medidas contínuas por mais anos.

Outros aspectos que devem ser considerados são apontados por Dalling et al. (2001)[44], em um estudo realizado com Alseis backiana, preliminarmente classificada como pioneira, apresenta algumas características de espécies tolerantes à sombra, como a permanência de um grande número de jovens no subosque com baixa taxa de mortalidade, decorrente de adaptações fisiológicas e morfológicas, incluindo eficiente interceptação luminosa, não sobreposição de folhas, tolerância a danos físicos e alta capacidade de rebrota. A convergência desses resultados indica que a classificação em grupos ecológicos não avalia toda a extensão possível de combinações dos atributos ecológicos das plântulas e indivíduos jovens encontrados nas florestas tropicais.

\section{CONCLUSÕES}

O grupo das espécies clímax de subosque apresentaram baixas taxas de mortalidade e apenas este grupo exibiu espécies com incremento populacional, condizente com a resposta esperada para espécies tolerantes à sombra. Já as espécies secundárias iniciais e clímax de dossel evidenciaram um contínuo de respostas, quanto à mortalidade e o recrutamento, indicando que os referidos grupos podem ocultar diferenças inter e intraespecíficas, quanto à tolerância à sombra.

Já o crescimento em altura e incremento em diâmetro não indicaram padrões para as espécies do mesmo grupo ou diferenças entre os mesmos. 


\section{REFERÊNCIAS BIBLIOGRÁFICAS}

1. Connell JH, Green PT. Seedling dynamics over thirty-two years in a tropical rain forest tree. Ecology. 2000 Feb;81(2):568-584, doi:10.1890/0012-9658(2000)081[0568:SDOTTY]2.0. CO;2.

2. Clark DA, Clark DB. Getting to the canopy: tree height growth in a neotropical rain forest. Ecology. 2001 May;82(5):1460-1472, doi:10.1890/0012-9658(2001)082[1460:GTTCTH]2.0.CO;2.

3. Whitmore TC. A review of some aspects of tropical rain forest seedling ecology with suggestions for further enquiry. In: Swaine MD (Ed.) Ecology of tropical forest tree seedlings. Man and the Biosphere Series Volume 17. UNESCO (Paris) and the Parthenon Publishing Group, Carnforth, UK, 1996, p.339.

4. Ramos FN, Matos DMS, Santos FAM. Spatial distribution of seeds and juveniles of Enterolobium glaziovii Bentham (Leguminosae, Mimosoideae) in the Atlantic Forest, Brazil. Acta Botanica Brasilica. 2005;19:609-614, dx.doi.org/10.1590/S0102-33062005000300022.

5. Nunes MH, Higuchi P, Silva AC, Van den Berg E, Santos MCN. Dinâmica de populações de espécies arbóreas em fragmentos de floresta aluvial no sul de Minas Gerais, Brasil. Floresta. 2016 Mar;46 (1):5766, doi://dx.doi.org/10.5380/rf.v46i1.34782

6. Karyati, Ipor IB, Jusoh I, Wasli ME. The diameter increment of selected tree species in a secondary tropical forest in Sarawak, Malaysia. Biodiversitas. 2017 Jan;18(1):304-311, doi:10.13057/biodiv/d180140.

7. Lieberman D, Hartshorn GS, Lieberman M., Peralta R. Forest dynamics at La Selva Biological Station, Costa Rica, 1969-1985. In: Gentry AH (Ed.). Four neotropical rainforests. New Haven and London:Yale University Press. 1990. p.509-521.

8. Nascimento HEM, Laurance WF, Condit R, Laurance SG, D`angelo S, Andrade AC. Demographic and life-history correlates for Amazonian trees. Journal of Vegetation Science. 2005 Dec;16(6):625-634, doi:10.1111/j.1654-1103.2005.tb02405.x

9. Appolinário V, Oliveira-Filho AT, Guilherme FAG. Tree population and community dynamics in a Brazilian tropical semideciduous forest. Revista Brasileira de Botânica. 2005 Apr/Jun;28(2):347-360, doi.org/10.1590/S0100-84042005000200014.

10. Finegan B, Camacho M, Zamora N. Diameter increment patterns among 106 tree species in a logged and silviculturally treated Costa Rica rain forest. Forest Ecology and Management. 1999 Aug;121(3):159-176, doi.org/10.1016/S0378-1127(98)00551-9.

11. Kariuki M, Rolfe M, Smith RGB, Vanclay JK, Kooyman RM. Diameter growth performance varies with species functional-group and habitat characteristics in subtropical rainforests. Forest Ecology and Management. 2006 Apr;225(1-3):1-14,doi.org/10.1016/j.foreco.2005.07.016.

12. Salami B, Higuchi P, Silva AC, Ferreira TS, Marcon AK, Buzzi F, Bento MA. Dinâmica de populações de espécies arbóreas em um fragmento de Floresta Ombrófila Mista Montana em Lages, Santa Catarina: Ciência Florestal. 2017;27(1):105-116, doi.org/10.5902/1980509826451.

13. Oliveira-Filho AT, Carvalho WAC, Machado ELM, Higuchi P, Appolinário V, Castro GC, Silva, AC, Santos RM, Borges LF, Corrêa BS, Alves JM. Dinâmica da comunidade e populações arbóreas da borda e interior de um remanescente florestal na Serra da Mantiqueira, Minas Gerais, em um intervalo de cinco anos (1999-2004). Revista Brasileira de Botânica. 2007 Jan/Mar;30(1):149-161, doi.org/10.1590/S0100-84042007000100015.

14. IBGE - Instituto Brasileiro de Geografia e Estatística. Manual Técnico da Vegetação Brasileira. Série Manuais Técnicos em Geociências 1, $2^{a}$ edição revista e ampliada. Rio de Janeiro: IBGE, 2012. 271 p.

15. Köppen W. Climatologia: con un estudio de los climas de la tierra. México: Fondo de Cultura Econômica; 1948. 478 p.

16. Rodrigues RR. 40 ha de Florestas do Estado de São Paulo: Uma Experiência Multidisciplinar. $4^{\circ}$ Relatório Científico. http://www.lerf.esalq.usp.br/parrel2005.php (acesso em 25-VIII-2016). 2006.

17. Soares-Junior AA, Vidal-Torrado P, Silva AC. Mapeamento ultra detalhado dos solos do Parque Estadual "Carlos Botelho" destinado ao Projeto Diversidade, Dinâmica e Conservação em Florestas do Estado de São Paulo: 40ha de Parcelas Permanentes. Escola Superior de Agricultura "Luiz de Queiroz", Piracicaba. 2004.Disponível em: http://www.lerf.eco.br/downloads/parcelas_permanentes_3o_relatorio_tematico_do_projeto parcel as_permanentes.pdf. (acesso em 20-IV-2016).

18. Muniz MRA. Estudo do regime de luz nas quatro principais formações fitogeográficas no estado de São Paulo durante o inverno do ano de 2003 [Dissertação de Mestrado], Campinas (SP): Universidade Estadual de Campinas, 2004. 189 p.

19. Lima RAF. Estrutura e regeneração de clareiras em Florestas Pluviais Tropicais. Revista Brasileira de Botânica. 2005 Oct/Dec;28(4):651-670, doi.org/10.1590/S0100-84042005000400002.

20. Krohne, D. General Ecology. Belmont: Thomson Brooks/Cole; 2001. 423 p. 
21. Brancalion PHS, Gandolfi S, Rodrigues RR. Restauração florestal. São Paulo: Oficina de Textos; 2015. $429 \mathrm{p}$.

22. Bernacci LC, Franco GADC, Arbocz CF, Catharino ELM, Durigan G, Metzger JP. O efeito da fragmentação florestal na composição e riqueza de árvores na região da reserva do Morro Grande (Planalto de Ibiúna, SP). Revista do Instituto Florestal. 2006 Dez;18:121-166.

23. Leite EC, Rodrigues RR. Fitossociologia e caracterização sucessional de um fragmento de Floresta Estacional no sudeste do Brasil. Revista Árvore. 2008;32(3):583-595, doi.org/10.1590/S010067622008000300019.

24. Gandolfi S. Estudo florístico e fitossociológico de uma floresta residual na área do aeroporto internacional de São Paulo, município de Guarulhos, SP. [Tese de mestrado] Campinas (SP):Universidade Estadual de Campinas; 1991. 232 p.

25. Souza FM, Cielo-Filho R, Ivanauskas NM, Franco GADC, Toniato MTZ, Matos IFA, Kanashiro MM, Aguiar OT, Baitello JB. A vegetação da Estação Ecológica de Xitué, Ribeirão Grande-SP: subsídios para o plano de manejo. IF Série Registros, 2014 Dez;51:5-30, doi.org/10.4322/ifsr.2014.001.

26. Catharino ELM, Bernacci LC, Franco GADC, Durigan G, Metzger JP. Aspectos da composição e diversidade do componente arbóreo das florestas da Reserva Florestal do Morro Grande, Cotia, SP. Biota Neotropica. 2006;6(2):1-28, doi.org/10.1590/S1676-06032006000200004.

27. Sztutman M, Rodrigues RR. O mosaico vegetacional numa área de floresta contínua da planície litorânea, Parque Estadual da Campina do Encantado, Pariquera Açú, SP. Revista Brasileira de Botânica. 2002 Jun;25(2):161-176.

28. Oliveira-Filho AT, Carvalho AC, Vilela EA, Curi N., Fontes AL. Diversity and structure of the tree community of a fragment of tropical secondary forest of the Brazilian Atlantic Florest domain 15 and 40 years after logging. Revista Brasileira de Botânica. 2004 Oct/Dec;27(4):685-701, doi.org/10.1590/S0100-84042004000400009.

29. Tabarelli M, Mantovani W. Colonização de clareiras naturais na floresta atlântica do Brasil. Revista Brasileira de Botânica. 1997 Jun;20(1):57-66.

30. Swaine MD, Lieberman D. Note on the calculation of mortality rates. Journal of Tropical Ecology. 1987 Aug;3:ii-iii.

31. R Core Team. R: The R Project for statistical computing. 2016. Disponível em https://www.rproject.org.

32. Lieberman D. Demography of tropical tree seedlings: a review. In: Swaine MD (Ed.). Paris: Parthenon Publishing Group; 1996. The ecology of tropical forest tree seedlings; p. 131-138.

33. Baker JP, Bunyavejchewin S. Suppression, release and canopy recruitment in five tree species from a seasonal tropical forest in western Thailand. Journal of Tropical Ecology 2006 Jul;22(5):521-529, doi.org/10.1017/S0266467406003312.

34. Nunes YR, Petrere Jr M. Structure and dynamics of a Cariniana estrellensis (Lecythidaceae) population in a fragment of Atlantic Forest in Minas Gerais, Brazil. Rodriguésia. 2012 Apr/Jun;63(2):257-267, doi=10.1590/S2175-78602012000200002\&pid=S2175-78602012000200002.

35. Condit R, Hubbell SP, Foster RB. Mortality rates of 205 neotropical tree and shrub species and the impact of a severe drought. Ecological Monographs. 1995 Feb;65(4):419-439, doi:10.2307/2963497.

36. Alvarez-Buylla ER, Martínez-Ramos M. Demography and allometry of Cecropia obtusifolia, a neotropical pioneer tree - an evaluation of the climax-pioneer paradigm for tropical rain forests. Journal of Tropical Ecology. 1992;80:275-290.

37. Howe HF. Survival and growth of juvenile Virola surinamensis in Panama: effects of herbivory and canopy closure. Journal of Tropical Ecology. 1990 Jul;6(3):259-280, doi.org/10.1017/S0266467400004508.

38. Nath CD, Dattaraja HS, Suresh HS, Joshi NV, Sukumar R. Patterns of tree growth in relation to environmental variability in the tropical dry deciduous forest at Mudumalai, southern India. J. Biosci. 2006 Dec;31(5):651-669. //www.ias.ac.in/article/fulltext/jbsc/031/05/0651-0669

39. Uhl C., Clark K, Maquirino P. Vegetation dynamics in amazonian treefall gaps. Ecology. 1988 Jun;69(3):751-763, doi:10.2307/1941024.

40. Clark DA, Clark DB. Análisis de la regeneración de árboles del dosel em bosque muy húmedo tropical: aspectos teóricos y prácticos. Revista de Biologia Tropical. 1987;35(supl.):41-54.

41. Fox JC, Ades PK, Bi HQ. Stochastic structure and individual-tree growth models. Forest Ecology and Management. 2001 Sep;154(3):261-276.

42. Brienen RJW, Zuidema PA, During HJ. Autocorrelated growth of tropical forest trees: Unraveling patterns and quantifying consequences. Forest Ecology and Management. 2006 Dec;237(1-3):179-190.

43. Davies SJ. Tree mortality and growth in 11 sympatric Macaranga species in Borneo. Ecology. 2001 Apr;82(4):920-932, doi:10.1890/0012-9658(2001)082[0920:TMAGIS]2.0.CO;2. 
44. Dalling JW, Winter K, Nason JD, Hubbell SP, Murawski DA, Hamrick JL. The unusual life history of Alseis blackiana: a shade-persistent pioneer tree? Ecology. 2001 Apr;82:933-945, doi:10.1890/00129658(2001)082[0933:TULHOA]2.0.CO;2. 